\title{
Foreword \\ Observing the mm Universe with the NIKA2 \\ camera
}

The "mm Universe @NIKA2" conference is the second edition in a series of biennial workshops. The first edition was held in Grenoble and organised by the LPSC (CNRS, Université Grenoble Alpes) in June 2019.

This international conference is dedicated to the scientific exploitation of the NIKA2 camera installed at the 30-m telescope of IRAM (Institut de Radio Astronomie Millimétrique) located in Pico Veleta (Spain) but it is open to the whole scientific community working on related science. NIKA2 is a millimetre camera, capable of surveying large areas of the sky at a high-angular resolution, with a high sensitivity and a large field of view. It was designed and built by the NIKA2 collaboration.

It has been installed in October 2015 at the IRAM 30-m telescope and commissioned in 2017. NIKA2 is open to the scientific community for observations for the next decade and provides key observations from the galactic scale to the high-redshift Universe. It allows observers to address questions, such as the environmental impact on dust properties, the star formation processes at low and high redshifts, the evolution of large-scale structures and the use of galaxy clusters for precision cosmology.

The conference covered theoretical, observational and instrumental topics related to the $\mathrm{mm}$ Universe, i.e. from stellar to cosmological scales.

The Conference was organised by the Department of Physics at Sapienza University in Rome and held online via Zoom due to the current situation. Luckily the virtual format allowed all the interested participants to be able to take part, even if the useful exchange of discussions was limited to dedicated Slack channels. The number of 
participants was 145 , from several countries. The conference lasted 5 days with 64 presentations in plenary sessions ${ }^{1}$.

We believe that it was a great success thanks to the feedback received, the enthusiasm of the participants and the high quality of their presentations. All the remote presentations and discussions went smoothly thanks to the great involvement of the chairpersons and the invaluable help of all the members of the Local Organising Committee. The organization of the conference was made possible thanks to funding from the Sapienza University: Finanziamenti per convegni, seminari, workshop - Classe A 2019 n. CC11916B7A19FBC7 and Progetti di Ricerca Medi 2019 n. RM11916B7540DD8D.

All proceedings articles in this volume have been peer-reviewed. Thanks are due to all referees and authors for their contributions to the editing process of the "mm Universe @NIKA2" conference proceedings.

M. De Petris (chair), A. Ferragamo and F. Mayet

Scientific editors of the 2 nd edition of the conference " $\mathrm{mm}$ Universe @ NIKA2"

${ }^{1}$ see https://agenda.infn.it/event/25056/ 